\title{
Accuracy and precision of the Tobii X2-30 eye-tracking under non ideal conditions
}

\author{
A. Clemotte ${ }^{1}$, M. Velasco ${ }^{1}$, D. Torricelli ${ }^{1}$, R. Raya ${ }^{1}$ and R. Ceres ${ }^{1}$ \\ ${ }^{1}$ Spanish National Council for Science Research (CSIC), Madrid, Spain \\ a.clemotte@alumnos.upm.es, \{miguel.velasco,diego.torricelli,rafael.raya,ramon.ceres\}@csic.es
}

Keywords: Remote eye trackers, Tobii X2-30, Spatial data quality, Accuracy, Precision

\begin{abstract}
This document describes a methodology for the measurement of accuracy and precision of a remote eye tracker, the Tobii X2-30, under non-ideal condition (without the use of a chinrest). The test was performed with 10 people (without disabilities). The results are: 2.46 and 1.91 degrees for the accuracy and precision respectively, while the device datasheet describe values of 0.40 and 0.26 degrees (accuracy and precision respectively). As expected, values calculated are higher, but they are better descriptors of the device's performance in conditions of normal use, where user can move the head freely. The results will be used to establish targets' size on the screen while the eye-tracker is used by people with cerebral palsy.
\end{abstract}

\section{INTRODUCTION}

This study is part of the InteraPCion project (InteraPCion project webpage, 2014), which seeks to design adapted interfaces for people with cerebral palsy (CP).

People with $\mathrm{CP}$ have trouble interacting with their environment. The technology allows decrease the difficulties of interaction with the environment through games or adapted therapies.

These people however are not able to use common computer access interfaces, such as keyboards, mouse or touchscreens. They need adapted access channels.

The characteristics of these users are: low postural control, especially for the extremities and, inability to speak. The eye-trackers systems are an interesting option, which has not been deeply explored yet in this context.

It is important that eye-tracking studies report the real accuracy and precision of the eye trackers (Blignaut and Beelders, 2012)(Akkil et al., 2014) It is argued that the values provided by the manufacturers are representative of the best possible capability of the eye tracker under ideal conditions and for participants with good tracking probabilities (Tobii X2-30 Eye Tracker Accuracy and Precision
Test Report, 2013)(Accuracy and Precision Test Method for Remote Eye Trackers, 2011).

To answer this question an experiment was carried out in order to identify the precision and accuracy of the Tobii x2-30 with non-disabled people, under non ideal conditions (without any chinrest). This information will be used to establish the minimum size of objects on the screen during the design of interfaces for people with disabilities.

\section{MATERIALS AND METHODS}

\subsection{Protocol test}

The protocol for the test was the following:

1. Check the eye-tracker position with respect to the screen. This must be positioned in the middle of the screen and both must be coplanar. If the eye-tracker is not well adjusted, an offset can be introduced in the measures.

2. Run the Tobii Studio (record software) (Tobii Studio software description webpage, 2014).

3. Take note of the user name, year of birth, gender, and issues related to his vision.

4. Explain to the user the test procedure. 
5. Check the user position with respect to the eye-tracker. He must be aligned with the eyetracker.

6. Calibrate the eye-tracker.

7. Start the test.

8. Rest for 5 minutes.

9. Calibrate the eye-tracker.

10. Start the test.

11. Check that the eye-tracker data was recorded correctly.

The test lies in:

1. The screen stays white for 1 second.

2. The first target showed up (Figure 1).

3. The user must look at the centre of the target 5 seconds

4. Then, the target changes its position automatically.

5. Repeat from the step 2,15 more times.

6. After 16 targets have been displayed, the test ends

Under ideal conditions, participants would use a chinrest in order to prevent any head movements that could affect the measurements. The aim of this study was to describe the performance of the eye tracking device under real and everyday conditions, where the user's head can freely move while he uses the eye tracker.

Nevertheless, in step five it was ensure that the user is within range of the eye tracker, according to the technical data.

\subsection{Target features}

The target consists in 2 concentric circles, with a 30 pixels (radius) internal black circle and a 100 pixels (radius) external red circle as depicted in Figure 1.

The target can be positioned in 16 positions, over 4 columns and 4 rows (Figure 2), according to the coordinates of Table 1.

The target position for each test was random, following the order depicted in the Table 2.

In total, each target is displayed during 5 seconds. Each test lasts: 5 seconds / target x 16 targets +1 initial second $=81$ seconds (approximately 1 minute and a half).

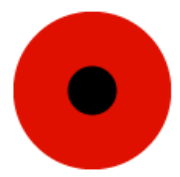

Figure 1. The target lies in two concentric circles, the internal black and the external red.

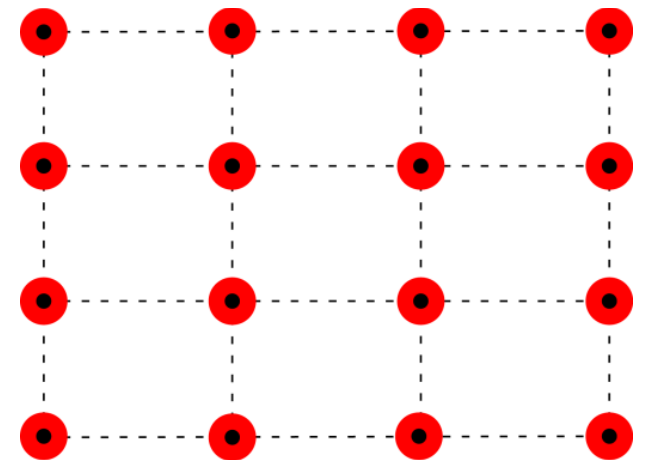

Figure 2. Targets are distributed in 4 rows and 4 columns. Origin of the coordinate is at top-left.

Table 1. Rows and columns' coordinates

\begin{tabular}{|c|c|}
\hline Row & Coordinate \\
\hline 1 & 100 \\
\hline 2 & 370 \\
\hline 3 & 655 \\
\hline 4 & 920 \\
\hline Column & Coordinate \\
\hline 1 & 100 \\
\hline 2 & 460 \\
\hline 3 & 820 \\
\hline 4 & 1180 \\
\hline
\end{tabular}

Table 2. Order of appearance of the targets, specified by it coordinates values.

\begin{tabular}{|c|c|c|}
\hline Order of appearance & Row & Column \\
\hline 1 & 1 & 3 \\
\hline 2 & 4 & 4 \\
\hline 3 & 4 & 1 \\
\hline 4 & 1 & 4 \\
\hline 5 & 1 & 1 \\
\hline 6 & 2 & 2 \\
\hline 7 & 3 & 1 \\
\hline 8 & 2 & 1 \\
\hline 9 & 3 & 3 \\
\hline 10 & 2 & 3 \\
\hline 11 & 4 & 2 \\
\hline 12 & 2 & 4 \\
\hline 13 & 3 & 4 \\
\hline 14 & 1 & 2 \\
\hline 15 & 4 & 3 \\
\hline 16 & 3 & 2 \\
\hline & & \\
\hline
\end{tabular}

\subsection{Eye-tracker features}

The eye-tracker used for the experiment was the Tobii X2-30 (Tobii X2-30 system description webpage, 2014), at a sampling frequency of $40 \mathrm{~Hz}$. This eye tracker is a remote system (not headmounted), able to collect data from both eyes (binocular). Accuracy and precision under ideal 
conditions are 0.4 and 0.26 degrees respectively, at a fixed distance from the user's head to the device. The eye tracker is able to operate at a distance between 40 to $90 \mathrm{~cm}$ to the user. The Tobii X2-30 allows head movements (at $70 \mathrm{~cm}$ ) of 50 and $36 \mathrm{~cm}$, in the horizontal and vertical axes, respectively (Tobii X2-30 Eye Tracker User's manual. Appendix A: Tobii X2-30 Eye Tracker Specifications, 2013)0. The Tobii run the firmware 1.0.324726.20130603.1649.root.

The experiment design was performed with the

Tobii Studio 3.2.1.190 Professional Edition.

\subsection{Computer features}

The computer used for the test was a HP Compaq 8000 , with the characteristics shown in Table 3.

Table 3. Features of the computer used for the experiment.

\begin{tabular}{|c|c|}
\hline Computer & HP Compaq 8000 Elite CMT \\
\hline CPU & Intel Core 2 Quad Q9500 \\
\hline CPU Speed & $2 \times 2.83 \mathrm{Ghz}$ \\
\hline RAM & 4GB \\
\hline OS & W7 Professional 32b \\
\hline Graphics card & GeForce 8500 GT \\
\hline
\end{tabular}

At the moment of the test, only the Tobii Studio software was running. Appearance and performance of Windows were set up to "best performance" and the energy saving options were set up to "hi performance".

\subsection{Screen features}

The screen used for the test was a HP L1950g 19inch, with the features described in the Table 4.

According to the screen characteristics, each pixel has $0.03 \mathrm{~cm}$. Then, the internal black circle has $0.9 \mathrm{~cm}(0.03 \mathrm{~cm} /$ pix X 30pix $)$ and the external red circle has $3 \mathrm{~cm}(0.03 \mathrm{~cm} /$ pix X 100pix $)$.

Table 4. Features of the screen used for the test.

\begin{tabular}{|c|c|}
\hline Screen & HP L1950g \\
\hline Dimensions & 19 inch $(48.25 \mathrm{~cm})$ \\
\hline Technology & LCD \\
\hline Screen Opening $(\mathrm{WxH})$ & $14.9 \times 11.9 \mathrm{inch}(37.8 \mathrm{x}$ \\
& $30.3 \mathrm{~cm})$ \\
\hline Aspect Ratio & $5: 4$ \\
\hline Resolution & $1280 \times 1024$ \\
\hline Pixel Pitch & $0.294 \mathrm{~mm}$ \\
\hline Frequency & $60 \mathrm{~Hz}$ \\
\hline
\end{tabular}

\subsection{Calibration procedure}

Five points were used for the calibration. The Calibration procedure is:

1. A small animated circle is shown on the screen to catch the user's attention.

2. When it arrives at the calibration point, the point rests for about 0.5 seconds to make the user stare at it. Then, the object shrinks to focus the gaze.

3. When shrunk, the eye tracker starts to collect data for the specific calibration point.

4. The eye tracker waits until calibration data from the current position is collected.

5. The circle is enlarged again.

6. Steps 1-5 are repeated for all 5 calibration points.

\subsection{Participants' information}

In total, 10 participants were recruited, whose characteristics are shown in the Table 5.

$40 \%$ of the participants are women, and the $60 \%$ men. All participants are regular readers. 3 out of the 10 participants (U2, U7 and U10) have had contact with an eye-tracker before. One user has systematic contact with an eye-tracker.

Half of the participants normally use glasses while working with a computer. These people have taken the tests without their glasses. One user uses contact lenses. This user has performed the test with their contact lenses on.

The color of the eyes of the participants as well the dominant eye is indicated in Table 6 . The user U10 has very narrow eye shape.

Table 5. Year of birth and gender of the participants.

\begin{tabular}{|c|c|c|}
\hline User & Year of birth & Sex \\
\hline U1 & $1970-1974$ & Female \\
\hline U2 & $1985-1989$ & Male \\
\hline U3 & $1985-1989$ & Male \\
\hline U4 & $1985-1989$ & Male \\
\hline U5 & $1990-1994$ & Female \\
\hline U6 & $1985-1989$ & Female \\
\hline U7 & $1980-1984$ & Male \\
\hline U8 & $1985-1989$ & Male \\
\hline U9 & $1980-1984$ & Female \\
\hline U10 & $1980-1984$ & Male \\
\hline
\end{tabular}


Table 6. Eyes color (B: Brown, G: Green), dominant eye (L: Left, R: Right) and issues related with the vision per user.

\begin{tabular}{|c|c|c|c|}
\hline User & Eyes color & Dominant eye & Vision \\
\hline U1 & B & R & Not Corrected \\
\hline U2 & B & R & Not Corrected \\
\hline U3 & G & L & Contact lens \\
\hline U4 & B & R & Glasses \\
\hline U5 & B & R & Glasses \\
\hline U6 & G & L & Glasses \\
\hline U7 & B & L & Not Corrected \\
\hline U8 & B & R & Glasses \\
\hline U9 & G & R & Not Corrected \\
\hline U10 & G & L & Glasses \\
\hline
\end{tabular}

\subsection{Environment illumination}

The illumination in the test room can be considered stable during the tests, which last approximately one hour. The illumination was sunlight and fluorescent lighting.

\subsection{Data pre-processing}

Data provided by the eye-tracker at each sample time are: the X,Y coordinates of the point where the participant is looking at (gaze of each eyes), and the $\mathrm{X}, \mathrm{Y}, \mathrm{Z}$ coordinates of the position of the eyes, as depicted in the Table 7 . There is also the target position coordinates in every sample time, defined by the protocol.

Table 7. Data provided by the eye-tracker in each sample data: gaze point and eye position. The target position at the screen is also known, defined by the protocol.

\begin{tabular}{|c|c|}
\hline $\begin{array}{c}\text { Left gaze } \\
(\mathrm{X}[\mathrm{t}], \mathrm{Y}[\mathrm{t}])\end{array}$ & $\begin{array}{l}\text { Right gaze } \\
(\mathrm{X}[\mathrm{t}], \mathrm{Y}[\mathrm{t}])\end{array}$ \\
\hline $\begin{array}{c}\text { Left eye } \\
\text { position } \\
(\mathrm{X}[\mathrm{t}], \mathrm{Y}[\mathrm{t}], \mathrm{Z}[\mathrm{t}])\end{array}$ & $\begin{array}{c}\text { Right eye } \\
\text { position } \\
\text { (X[t],y[t], }[t])\end{array}$ \\
\hline $\begin{array}{r}\text { Targe } \\
\text { (X }\end{array}$ & $\begin{array}{l}\text { ition } \\
\text { t]) }\end{array}$ \\
\hline
\end{tabular}

The origin of the coordinate system is the upper left corner of the screen, then the coordinate of the $\mathrm{Z}$ axis is zero constantly for the target and gaze positions (Figure 3).

For each sample provided by the eye-tracker a number of simplification were made. One gaze point is calculated (Gaze), with the average of each eye gaze coordinates independently:

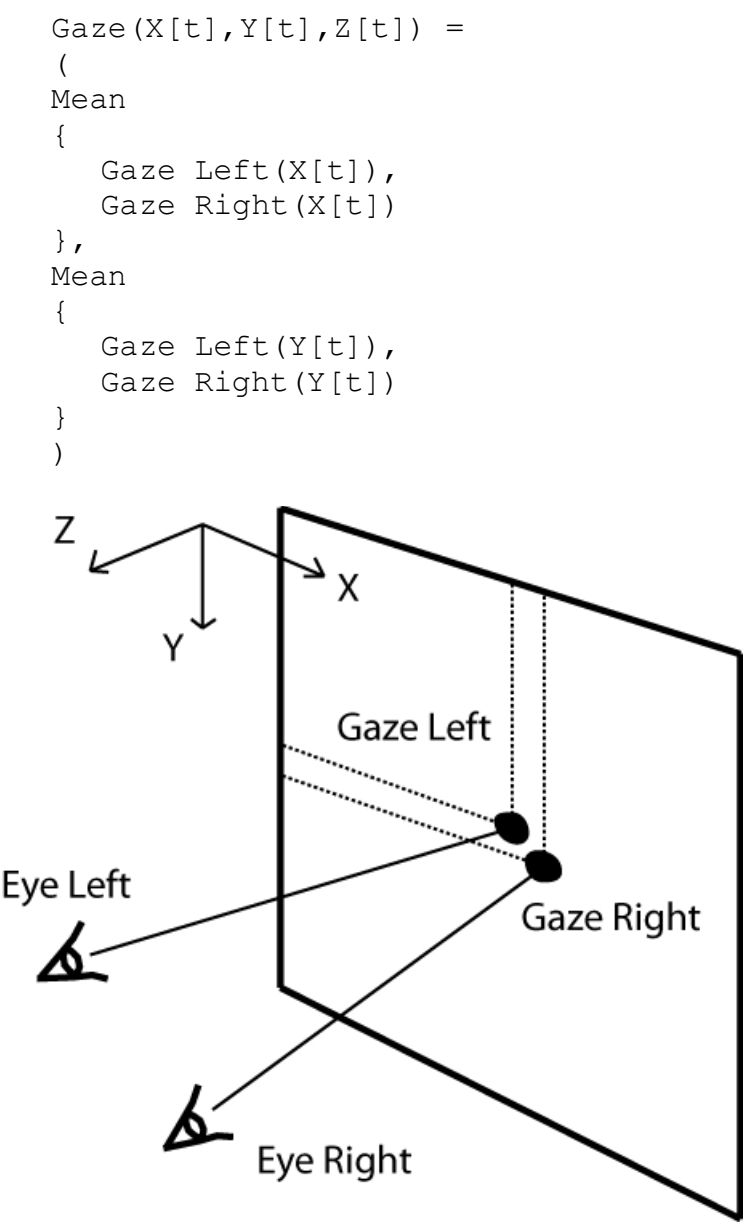

Figure 3. This figure depicted the gaze for each eye projected to the screen as a red and blue line. Also are depicted the points of intersection between the gaze and the screen (red and blue circles). Coordinates origin is the upper left corner of the screen.

Also, for each sample provided by the eyetracker, one eye position is calculated (Eye), with the average of both eye coordinates:

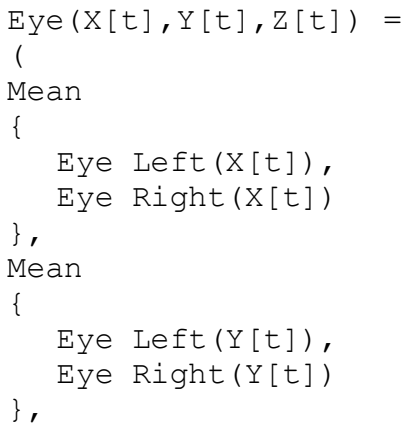




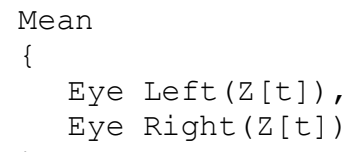

Summarizing, after the simplification the data are the presented in the Table 8, depicted graphically in the Figure 4.

Table 8. After the simplification, the data are the average Gaze point, Eye position and Target position.

\begin{tabular}{|c|c|}
\hline Gaze & $(\mathrm{X}[\mathrm{t}], \mathrm{Y}[\mathrm{t}], 0)$ \\
\hline Eye position & $(\mathrm{X}[\mathrm{t}], \mathrm{Y}[\mathrm{t}], \mathrm{Z}[\mathrm{t}])$ \\
\hline Target position & $(\mathrm{X}[\mathrm{t}], \mathrm{Y}[\mathrm{t}], 0)$ \\
\hline
\end{tabular}

With these three points (Gaze, Target and Eye position) two vectors are created: Eye-Target and Eye-Gaze:

$$
\begin{aligned}
& \mathrm{a}=\text { Eye - Target } \\
& \mathrm{b}=\text { Eye - Gaze }
\end{aligned}
$$

For each sample data the angle between these two vectors are calculated as follow:

$$
\begin{aligned}
\operatorname{Angle}(a, b)= & \operatorname{atan}(\operatorname{norm}( \\
& \operatorname{cross}(a, b)), \operatorname{dot}(a, b))
\end{aligned}
$$

Where atan calculate the inverse tangent, norm calculate the norm of the vector, cross calculate the cross product and dot calculate the dot product. With these angles, the accuracy and precision are calculated, as is defined later.

For the analysis, the last 4.25 seconds, after the target appeared, have been processed. The first 0.75 seconds are not used for the analysis because it is considered the time required to fix the gaze in the target displayed. The Figure 5 depict the first 0.75 seconds as a gray area.

Angles with a value greater than 3 times the standard deviation of all the angles are considered outliers and are discarded from further analysis.

\subsection{Definitions}

The accuracy and precision are calculated as the mean and standard deviation respectively of all the angles recorded (angles between the vectors EyeTarget and Eye-Gaze):

$$
\begin{aligned}
& \text { Accuracy }=\text { mean (angle) } \\
& \text { Precision }=\text { std (angle) }
\end{aligned}
$$

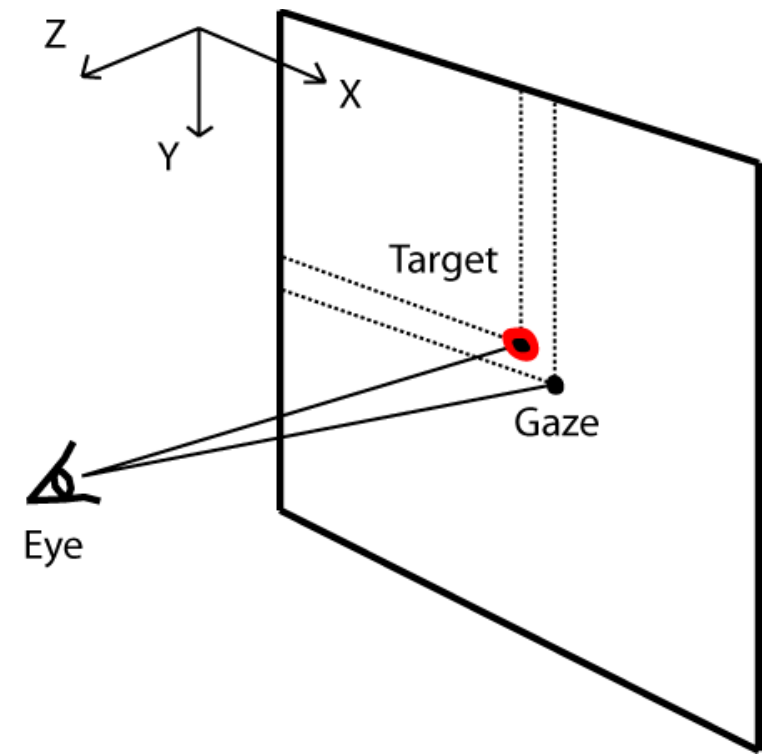

Figure 4. Graphically, the data simplified are the Target Gaze and Eye position.

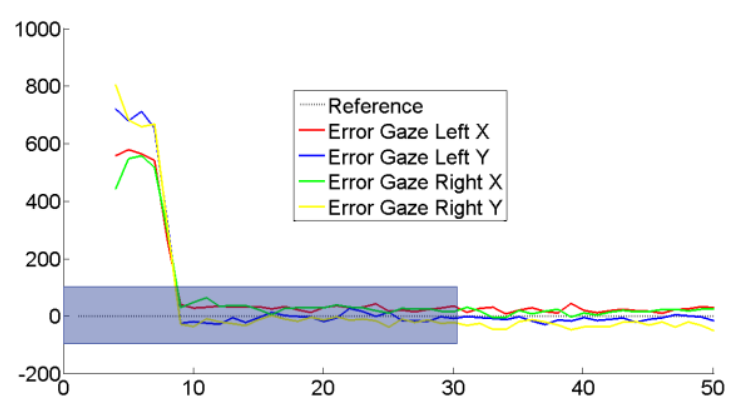

Figure 5. This figure depicted the error (gaze coordinate minus the target coordinate) as a function of time. In this figure the gray rectangle enclose the first 0.75 seconds not used for the analysis because it is considered the time required to fix the gaze in the target displayed.

\section{RESULTS}

After data preprocessing, the rate of outliers is $1.20 \%$ from a total of 44517 samples. Accuracy and precision without outliers are presented in the Table 9.

The histogram of Gaze is showed in the Figure 6 (as a heat-map), and histogram of the angles in the Figure 7. Both of these histograms were made after data pre-processing (without outliers). 
Table 9. Accuracy and precision of the data provided by the test and by the datasheet (Tobii X2-30 Eye Tracker User's manual. Appendix A: Tobii X2-30 Eye Tracker Specifications , 2013), in degrees.

\begin{tabular}{|c|c|c|}
\hline & Measured & Datasheet \\
\hline Accuracy & $2.46^{\circ}$ & $0.40^{\circ}$ \\
\hline Precision & $1.91^{\circ}$ & $0.26^{\circ}$ \\
\hline
\end{tabular}

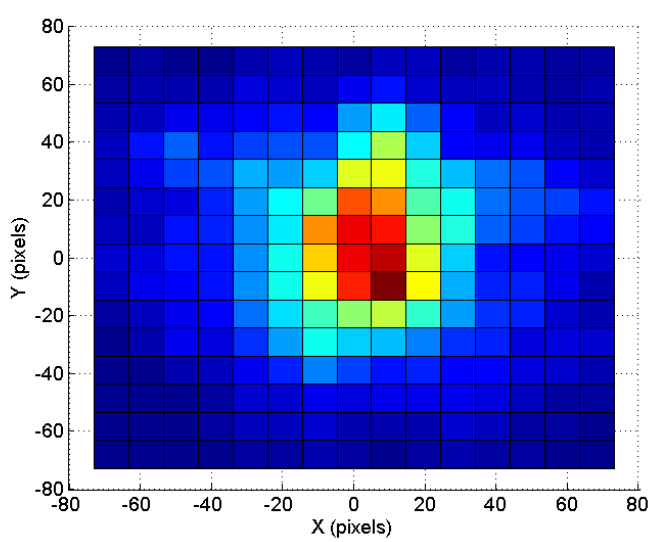

Figure 6. Histogram of the data recorded as a function of the $\mathrm{X}, \mathrm{Y}$ coordinates, in pixels.

Figure 8 shows the average gaze position for each target. Each target has associated 20 red points, representing each user (each participant perform 2 tests). For this figure, data with outliers were used.

\section{DISCUSSION}

The obtained results facilitated the establishment of the target size on the screen, according to the following criteria. The precision establish the minimum size of the target. Otherwise, the user of the eye-tracker cannot maintain the gaze over the target. Precision can be reduced filtering the signal.

The accuracy is more critical than the precision, because it cannot be reduced with filtering technics.

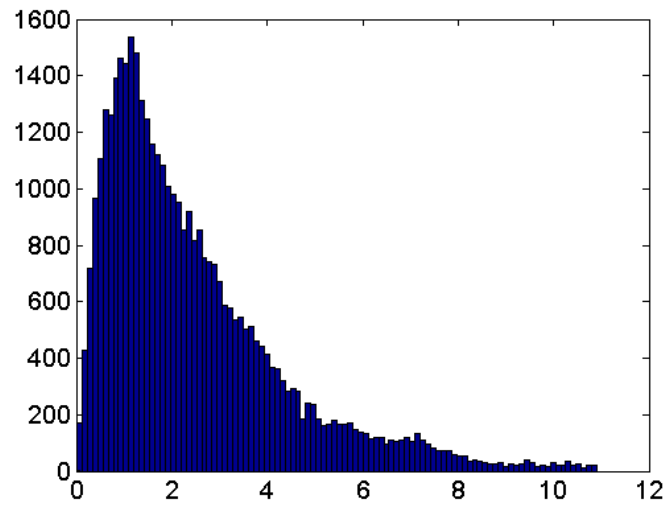

Figure 7. Histogram of the angles between the vectors Eye-Gaze and Eye-Target. The pick is at 1.15 degrees.

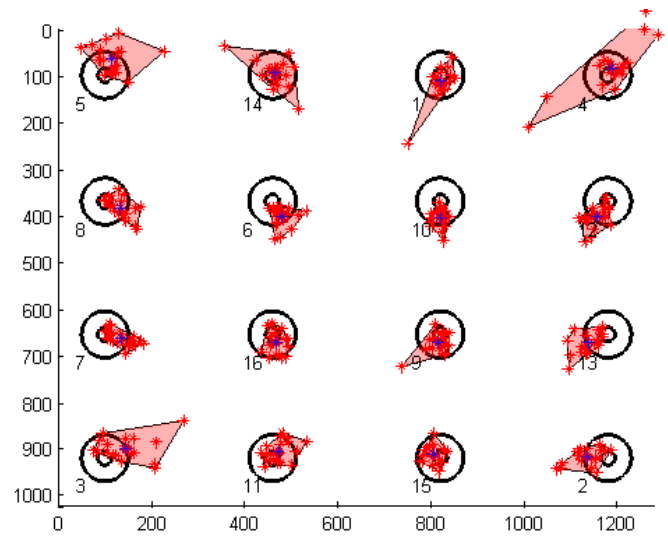

Figure 8. Average gaze position for each target. The axis measurement units are pixels.

\section{CONCLUSIONS}

We describe a method for the measurement of accuracy and precision of a remote eye tracker, the Tobii X2-30. The test was performed with 10 people, under non-ideal condition (without a chinrest). Even without the chinrest device, the user's head was always within the range of the eye tracker during the sessions.

The experiment outputs are 2.46 and 1.91 degrees for the accuracy and precision, respectively. As expected, this values are higher than the ones described by the device developers under ideal conditions, but might be better descriptors of the device's performance since were calculated in real conditions. 
All the participants in the study were young healthy subjects with perfect postural and motor control. The interest of this analysis is to expand the results to a population of disabled people, through the developed of alternative computer access. The results could be used to better define some parameters of alternative computer interfaces for disabled people, such as button size on the screen and distance between buttons.

\section{REFERENCES}

Tobii Studio software description webpage. Retrieved August, 2014, from http://www.tobii.com/en/eyetracking-research/global/products/software/tobiistudio-analysis-software/.

InteraPCion project webpage. Retrieved August, 2014, from http://www.car.upm-csic.es/bioingenieria/GBIO$\mathrm{CP} /$ proyectos.html.

Tobii X2-30 system description webpage. Retrieved August, 2014, from http://www.tobii.com/de/eyetracking-research/global/products/hardware/tobii-x230-eye-tracker/.

Blignaut, P., \& Beelders, T., 2012. TrackStick : A Data Quality Measuring Tool for Tobii Eye Trackers. In Proceedings of the Symposium on Eye Tracking Research and Applications - ETRA '12 (p. 293). New York: ACM Press.

Akkil, D., Isokoski, P., Kangas, J., Rantala, J., \& Raisamo, R. 2014. TraQuMe: A Tool for Measuring the Gaze Tracking Quality. In Proceedings of the Symposium on Eye Tracking Research and Applications ETRA'14 (pp. 327-330). New York, USA: ACM Press.

Tobii X2-30 Eye Tracker Accuracy and Precision Test Report. Version 1.0.1, 2013. Tobii Technology AB. Retrieved August, 2014, from http://www.tobii.com/Global/Analysis/Training/Metri cs/Tobii 20X2-30 Eye Tracker Accuracy and Precision Test Report.pdf?epslanguage $=$ de

Accuracy and Precision Test Method for Remote Eye Trackers. Version 2.1.1, 2011. Tobii Technology AB. Retrived August, 2014, from http://www.tobii.com/Global/Analysis/Training/Metri cs/Tobii_Test_Specifications_Accuracy_and_Precisio nTestMethod_version 2_1_1_.pdf

Tobii X2-30 Eye Tracker User's manual. Appendix A: Tobii X2-30 Eye Tracker Specifications. Version 1.0.1, 2013. Tobii Technology AB. Retrieved August, 2014 , from http://www.tobii.com/Global/Analysis/Downloads/Us er_Manuals_and_Guides/Tobii_X2-

30_EyeTrackerUserManual_WEB.pdf?epslanguage $=\mathrm{d}$ e 